\title{
Using the orbiting companion to trace WR wind structures in the 29d WC8d + O8-9IV binary CV Ser
}

\author{
Alexandre David-Uraz ${ }^{1}$ and Anthony F. J. Moffat ${ }^{1}$ \\ ${ }^{1}$ Département de Physique, Université de Montréal \\ C.P. 6128, Succursale Centre-Ville, Montréal, QC H3C 3J7, Canada \\ emails: alexandre@astro.umontreal.ca, moffat@astro.umontreal.ca
}

\begin{abstract}
We have used continuous, high-precision, broadband visible photometry from the MOST satellite to trace wind structures in the WR component of CV Ser over more than a full orbit. Most of the small-scale light-curve variations are likely due to extinction by clumps along the line of sight to the $\mathrm{O}$ companion as it orbits and shines through varying columns of the WR wind. Parallel optical spectroscopy from the Mont Megantic Observatory is used to refine the orbital and wind-collision parameters, as well as to reveal line emission from clumps.
\end{abstract}

Keywords. stars: Wolf-Rayet, stars: winds, outflows

\section{Introduction}

The primary aim of our project was to probe the structures in the wind of the WR component in the CV Ser WR + O binary by using high-precision photometry. The basic idea is shown in fig. 1. At different phases, clumps with varying sizes will go through the O star's line of sight, thus producing random dips in the light curve. We can then analyze these dips to find constraints on the sizes and shapes of the clumps.

However, in early studies ( Hjellming \& Hiltner (1963), Stepień (1970), Kuhi \& Schweizer (1970), Cowley et al. (1971)), CV Ser has proved to be a misbehaving binary system, with the depth of its eclipse varying with time. It was even reported to have stopped eclipsing. The most plausible explanation is that the wind of the dust-forming Wolf-Rayet component changed its structure between observations. However, for the first time, we show evidence for two consecutive eclipses with different depths, which might suggest a rapidly varying mass-loss rate.

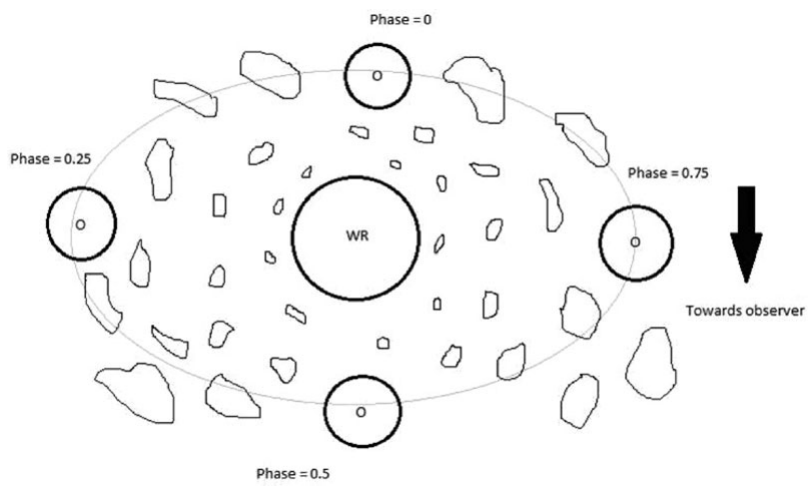

Figure 1. Intuitive model of the effects of clumps on the light curve. 


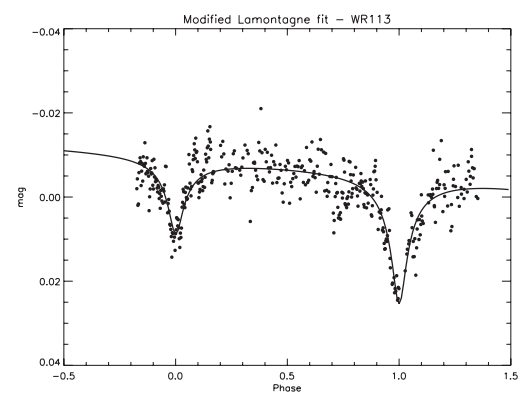

Figure 2. Best fit to the modified Lamontagne et al. model.

\section{Preliminary results}

We tried to fit our light curve using a first order model for atmospheric eclipses, as described in Lamontagne et al. (1996):

$$
\Delta m=\Delta m_{0}+A\left(\frac{\pi / 2+\arcsin \epsilon}{\sqrt{1-\epsilon^{2}}}\right)
$$

(with $\epsilon=(\sin i) \cos 2 \pi \phi, A=\frac{(2.5 \log e) k}{\left(I_{W} R / I_{O}\right)}$ and $k=\frac{\alpha \sigma_{e} \dot{M}}{4 \pi m_{p} v_{\infty} a}$.)

However, since our two eclipses do not have the same depth, it was necessary to make a small adjustment. We let $k$ vary linearly with time, which could indicate a change in the mass loss parameters (most probably $\dot{M}$ and/or $v_{\infty}$ ). We also had to allow $\Delta m_{0}$ to vary linearly with time, most likely due to detector drift. The best fit is shown in fig. 2 .

Assuming the values given in Lamontagne et al. (1996) for most parameters for CV Ser except $\dot{M}$, the fit yields a mass-loss rate of about $2.9 \cdot 10^{-5} M_{\odot} /$ year for the first eclipse. If we assume that the variation of the depth of the eclipse is entirely due to the variation of the mass-loss rate, we then find that the Wolf-Rayet mass-loss rate increases by $70 \%$ from the first eclipse to the second eclipse!

\section{What's next?}

There is still much to be done with CV Ser. First, Fourier and wavelet analyses will help detect coherent or random variations in the light curve (which can hopefully be linked to clumps). The spectra of both components of the binary system can be separated using the "shift and add" method. We will also check for changes in the spectra which could be linked to variations in the properties of the WR wind (mass-loss rate, temperature, terminal velocity, ionisation, etc.). Lührs' model can be applied to the excess emission to model the shock cone and compare the value of $i$ obtained to the one given by Lamontagne's model. Ultimately, the goal of this project is to characterize the clumping phenomenon. New data (MOST and ground-based spectra) taken during summer 2010 will help us complete our analysis.

\section{References}

Cowley, A. P., Hiltner, W. A., \& Berry, C. 1971, A\&A, 11, 407

Hjellming, R. M. \& Hiltner, W. A. 1963, ApJ, 137, 1080

Kuhi, L. V. \& Schweizer, F. 1970, ApJ (Letters), 160, L185

Lamontagne, R., Moffat, A. F. J., Drissen, L., \& Robert, C. et al. 1996, AJ, 112, 2227

Stȩpień, K. 1970, AcA, 20, 13 